\title{
Evaluación de 200 pacientes pediátricos expuestos al riesgo de contraer rabia
} Assessment of 200 pediatric patients exposed to rabies risk

\author{
Dr. Ali Osman Koksal ${ }^{a}$, Dra. Aslihan Arasli Yilmaz ${ }^{a}$, Dr. Osman Ozdemir ${ }^{a}$, \\ Dra. Seyma Cicek y Dra. Serife Yilmaz
}

\section{RESUMEN}

Antecedentes/Objetivo: La rabia continúa siendo un grave problema de salud pública, especialmente en los países subdesarrollados o en vías de desarrollo. El objetivo de este estudio fue investigar las características demográficas y los programas de vacunación de los pacientes ingresados con diagnóstico presuntivo de rabia al Centro de Emergencias Pediátricas de nuestro hospital, que funciona como uno de los centros de vacunación antirrábica de nuestra provincia. Métodos: En este estudio, se analizaron retrospectivamente las historias clínicas de 200 pacientes ingresados al Centro de Emergencias Pediátricas con presunta exposición al virus de la rabia.

Resultados: Entre los 200 casos, se halló que el riesgo de contraer rabia era mayor en el grupo de 5 a 9 años. El $68,5 \%$ de los casos tenían antecedentes de haber sido mordidos por un perro; el 29,5\%, de haber sido rasguñados por un gato; y el $2 \%$, de haber tenido contacto con otros animales. En el $76 \%$ de los casos se trataba de animales callejeros, solamente el 11\% tenían dueño y habían sido vacunados, y estaban bajo supervisión. Se administró solamente la vacuna antirrábica al 42,5\% de los pacientes ingresados, la vacuna antirrábica y la vacuna antitetánica al $51,5 \%$, y la vacuna antirrábica, la vacuna antitetánica y concentrado de inmunoglobulinas antirrábicas al 6\%. Se detectó que la profilaxis postexposición se había realizado según las recomendaciones en el $83,5 \%$ de los casos.

Conclusión: La rabia continúa siendo un problema de salud pública grave en los países en vías dedesarrollo, comoelnuestro.Consideramos que es necesario sensibilizar a la sociedad; las autoridades locales deben esforzarse por luchar contra los animales callejeros y supervisar los servicios; el personal de la salud involucrado debe actualizarse mediante capacitaciones para reducir los casos de rabia.

Palabras clave: niño; países en vías de desarrollo; profilaxis; rabia; vacunación.

http:/ /dx.doi.org/10.5546/aap.2015.419

\section{INTRODUCCIÓN}

La rabia es una infección viral aguda y mortal que afecta el sistema nervioso central. El virus causal de la rabia es un retrovirus neurotrópico del género Lyssavirus y de la familia
Rhabdoviridae. ${ }^{1}$ El virus tiene la capacidad de infectar a todos los animales de sangre caliente, y produce una infección que causa encefalitis mortal en casi todos los casos. La infección suele aparecer como resultado de la mordedura de un animal a un ser humano; sin embargo, se han informado casos después de un trasplante de órganos, o contacto con una herida en la piel y las mucosas. ${ }^{2}$

Según los datos de la Organización Mundial de la Salud (OMS) de 2010, se producen casos de rabia en más de 150 países de todo el mundo, y cada año mueren 55000 personas por esta infección. El 40\% de los seres humanos mordidos por un animal presuntamente rabioso son niños menores de 15 años. Cada año, se administra profilaxis postexposición a más de 15 millones de personas; este procedimiento previene 327000 muertes relacionadas con la rabia cada año. ${ }^{3}$ La rabia, que es un problema de salud pública importante en Turquía, constituye una enfermedad de denuncia obligatoria. Asimismo, nuestro país es uno de los tres países europeos con las tasas más altas de rabia en animales domésticos. ${ }^{4}$

El objetivo de este estudio fue investigar las características demográficas y los programas de vacunación de los casos expuestos a la rabia ingresados al Consultorio de Emergencias Pediátricas de nuestro hospital, que funciona como uno de los centros de vacunación antirrábica de nuestra provincia.

\section{MÉTODOS}

En nuestro estudio, se analizaron retrospectivamente los registros de los casos ingresados al Consultorio de 
Emergencias Pediátricas de nuestro hospital entre enero y diciembre de 2012 debido al riesgo de contacto con la rabia. Los criterios de inclusión del análisis fueron riesgo de contacto con la rabia a través de animales como gatos, perros y otros. Los datos se obtuvieron a partir del análisis del tipo de contacto o presunta exposición al virus de la rabia según la Directiva de Prevención y Control de la Rabia. Se evaluaron las características demográficas de los casos, la estación del año en que ocurrió el evento, el período hasta el ingreso al centro de salud después de haber tenido contacto con el animal, el tipo de lesión, la localización de la lesión, si se trataba de un animal callejero o con dueño o bajo supervisión, si el animal estaba o no vacunado, si se había administrado profilaxis para la rabia, los métodos de tratamiento y si estos fueron adecuados o no. Los casos se distribuyeron en grupos etarios de la siguiente manera: de 1 a 12 meses, de 1 a 4 años, de 5 a 9 años, de 10 a 14 años y de 15 a 18 años. El análisis estadístico de los datos se realizó con el programa SPSS 16.0. Los datos descriptivos se expresaron como frecuencia y porcentaje. Las diferencias entre los grupos, tal como edad, sexo, lugar de residencia, estación del año, tipo de animal, estado del animal y tipo de contacto, se evaluaron mediante la prueba $\chi^{2}$. Un valor de $p$ inferior a 0,05 se aceptó como estadísticamente significativo.

La adecuación de la profilaxis para la rabia se evaluó según la Directiva de Prevención y Control de la Rabia, publicada por el Ministerio de Salud, Dirección General de Servicios de Salud Básicos (del 9 de mayo de 2001, número B100TSH110002/7755) y revisada en $2005 .{ }^{5}$ En el último año, si el animal no había recibido la vacuna antirrábica (confirmación mediante carnet de vacunación), se administró la vacuna antirrábica a todos los animales con presunta rabia. En nuestro estudio, se administraron concentrado de inmunoglobulinas a los pacientes con heridas profundas o sucias, y la vacuna antirrábica según los criterios precedentes. El concentrado de inmunoglobulinas se inyectó, en todos los casos, en la herida y alrededor de esta.

\section{RESULTADOS}

En total, ingresaron 200 pacientes de entre $0 \mathrm{y}$ 18 años al Consultorio de Emergencias Pediátricas en 2012 debido al contacto de riesgo con la rabia. Todos los casos se incluyeron en el estudio. De estos, el 63\% (126) eran niños y el 37\% (74), niñas. La media de edad era 9,52 $\pm 4,38$ años. Cuando se los evaluó según los grupos etarios, se halló que el contacto de riesgo con la rabia era mayor (32\%) entre los 5 y los 9 años. Las características demográficas se indican en detalle en la Tabla 1. Se halló una diferencia estadísticamente significativa entre los grupos en cuanto a edad y sexo ( $p$ $<0,05)$. Entre los casos, 196 (98\%) provenían de áreas urbanas y 4 (2\%), de áreas rurales. Mientras que el invierno fue la estación durante la cual hubo menos ingresos (13\%), en el verano se produjo un pico de ingresos $(34,5 \%)$; esta diferencia fue estadísticamente significativa $(p<0,05)$. Entre los casos, $116(58 \%)$ sufrieron una mordedura, $80(40 \%)$ un rasguño, y $4(2 \%)$ tuvieron contacto a través de una herida abierta.

La evaluación de los casos por localización de lesión indicó que 106 (53\%) lesiones afectaban las extremidades superiores. Las lesiones en las extremidades inferiores, el tronco y el cuello y la cabeza correspondieron a $53(26,5 \%), 28(14 \%), y$ $13(6,5 \%)$ casos, respectivamente. La mordedura de un animal como tipo de lesión y las lesiones

TABLA 1. Características demográficas de los casos

\begin{tabular}{|c|c|c|}
\hline Características & $\mathbf{n}$ & $\%$ \\
\hline \multicolumn{3}{|l|}{ Grupos etarios } \\
\hline De 1 a 12 meses & 2 & 1 \\
\hline De 1 a 4 años & 25 & 12,5 \\
\hline De 5 a 9 años & 64 & 32 \\
\hline De 10 a 14 años & 63 & 31,5 \\
\hline De 15 a 19 años & 46 & 23 \\
\hline \multicolumn{3}{|l|}{ Sexo } \\
\hline Niñas & 74 & 37 \\
\hline Niños & 126 & 63 \\
\hline \multicolumn{3}{|l|}{ Lugar de residencia } \\
\hline Urbana & 196 & 98 \\
\hline Rural & 4 & 2 \\
\hline \multicolumn{3}{|l|}{ Estación } \\
\hline Primavera & 50 & 25 \\
\hline Verano & 69 & 34,5 \\
\hline Otoño & 55 & 27,5 \\
\hline Invierno & 26 & 13 \\
\hline \multicolumn{3}{|l|}{ Tipo de animal } \\
\hline Perro & 137 & 68,5 \\
\hline Gato & 59 & 29,5 \\
\hline Otro & 4 & 2 \\
\hline \multicolumn{3}{|l|}{ Estado del animal } \\
\hline Callejero/desconocido & 152 & 76 \\
\hline Con dueño/vacunado & 22 & 11 \\
\hline Con dueño/no vacunado & 24 & 12 \\
\hline Muerto/sacrificado & 2 & 1 \\
\hline \multicolumn{3}{|l|}{ Tipo de contacto } \\
\hline Mordedura & 116 & 58 \\
\hline Rasguño & 80 & 40 \\
\hline Contacto con una herida abierta & 4 & 2 \\
\hline
\end{tabular}


en las extremidades superiores como localización de la lesión fueron estadísticamente diferentes ( $p$ $<0,05)$ de otros tipos de lesiones o localizaciones.

El $68,5 \%$ de los casos tenían antecedentes de haber sido mordidos por un perro; el 29,5\%, de haber sido rasguñados por un gato; y el $2 \%$, de haber tenido contacto con otros animales. No se observaron antecedentes de lesión causada por animales salvajes. En el 76\% de los casos se trataba de animales callejeros, solamente el $11 \%$ de estos tenían dueño, habían sido vacunados y estaban bajo supervisión. Además, si bien el 12\% de los animales tenían dueño, no habían sido vacunados y estaban bajo supervisión, el 1\% estaban muertos o habían sido sacrificados. Se observó una diferencia estadísticamente significativa en el contacto o la presunta exposición respecto del tipo de animal $(p<0,05)$.

El momento del ingreso al servicio de emergencias después del contacto se produjo dentro de las 24 horas en el $61,5 \%$ de los casos, entre las 24 y 48 horas en el 13\%, entre 2 y 5 días después en el $14,5 \%$, y más de 5 días después en el $11 \%$ (Tabla 2). No fue posible hallar una diferencia estadísticamente significativa entre la edad y el momento del ingreso $(p>0,05)$.

Se administró solamente la vacuna antirrábica al $42,5 \%$ de los casos, la vacuna antirrábica y la vacuna antitetánica al 51,5\%, y la vacuna antirrábica, la vacuna antitetánica y concentrado de inmunoglobulinas antirrábicas al $6 \%$. Se administraron cinco dosis de vacuna al $85 \%$ de los casos, y tres dosis al $15 \%$. La profilaxis postexposición se consideró adecuada en el 83,5\% de los casos. En ningún caso se produjo anafilaxia. Ningún caso desarrolló la enfermedad ni falleció debido a la rabia.

\section{DISCUSIÓN}

A diferencia de estudios previos realizados en Turquía, este estudio es importante ya que es el primero en el cual se evaluaron los casos de riesgo de contraer rabia solamente en el

TABla 2. Momento de ingreso al servicio de emergencias después del contacto

\begin{tabular}{lcc}
\hline Período de ingreso & n & \% \\
\hline Primeras 24 horas & 123 & 61,5 \\
Entre 24 y 48 horas & 26 & 13 \\
Entre 2 y 5 días & 29 & 14,5 \\
Después de 5 días & 22 & 11 \\
\hline
\end{tabular}

grupo pediátrico. Özsoy, et al. detectaron que la rabia era más frecuente en el grupo de 5 a 14 años; sin embargo, cada grupo era sensible a las mordeduras de animales; por otro lado, la exposición se redujo en el grupo de 0 a 5 años. ${ }^{6}$ En nuestro estudio, la exposición fue más frecuente en el grupo de 5 a 9 años, y menor entre los 0 y los 12 meses. Consideramos que este resultado surge a partir del desarrollo psicosocial de los niños y su preferencia de jugar fuera de la casa a esa edad, lo cual aumenta el riesgo de contacto con animales callejeros.

En la bibliografía se informa que el riesgo de contacto con la rabia es menor en las áreas rurales en comparación con las áreas urbanas. ${ }^{7-9}$ De manera similar, el 98\% de los casos ingresados provenían de áreas urbanas, y esta fue una diferencia estadísticamente significativa en nuestro estudio. La mayor cantidad de casos provenientes de áreas urbanas indica que el control de los animales callejeros es más difícil en las provincias, que las medidas de las autoridades locales son insuficientes y que las personas de las áreas rurales no han desarrollado la conducta de solicitar atención en un centro de vacunación antirrábica.

El análisis del contacto con la rabia respecto de la estación del año indicó que se informaron casos con mayor frecuencia durante el verano en dos estudios diferentes realizados en Estambul. ${ }^{10,11}$ Los casos de contacto de riesgo con la rabia aumentan durante el verano porque los niños pasan más tiempo jugando al aire libre, ya que no asisten a la escuela y están de vacaciones. En este estudio también se demostró que el contacto con la rabia fue más frecuente durante el verano. Tomar medidas más estrictas contra el contacto con la rabia en el verano permitiría reducir la cantidad de casos. A tal fin, debería ofrecerse capacitación pública en forma regular y controlar a los animales callejeros, en especial durante el verano. Además podrían priorizarse los estudios sobre vacunación.

Si bien la infección por rabia se produce a través de la mordedura de animales callejeros en los países en vías de desarrollo, la fuente de la rabia en las personas de los países desarrollados son los animales salvajes. ${ }^{12}$ En todo el mundo, los animales considerados como fuente de rabia son perros $(91 \%)$, gatos $(2 \%)$, otras mascotas $(3 \%)$, murciélagos $(2 \%)$, otros animales salvajes $(<1 \%) .{ }^{13}$ En un estudio realizado en Angora, los casos de mordedura notificados estaban relacionados con perros $(68 \%)$ y gatos $(25 \%) .{ }^{5}$ 
El contacto con perros fue el más frecuente en los casos humanos con diagnóstico de rabia en Turquía entre 1992 y 2007. ${ }^{14}$ Los resultados de nuestro estudio coincidieron con la bibliografía. Se halló que el contacto con perros era significativamente más frecuente en comparación con el contacto con otros animales. Además, si se considera que dos tercios de nuestros casos fueron causados por animales salvajes y que el $98 \%$ de todos los ingresos son de áreas urbanas, podemos concluir que las autoridades locales no han logrado controlar los animales callejeros de manera adecuada, no se esfuerzan esmeradamente en relación con este tema y no se cumplen los mecanismos de supervisión.

Se ha detectado que los roedores pequeños, como las ardillas, los hámsters, los conejillos de Indias, las tamias, las ratas y los ratones, y los animales como los conejos y las liebres no causan rabia en los seres humanos. No se hallaron resultados positivos para rabia en más de 1000 ratones domésticos en estudios realizados en los últimos 20 años. Por lo tanto, no es necesario administrar profilaxis antirrábica cuando se tuvo contacto con estos animales. ${ }^{15}$ En nuestro estudio, consideramos que no se administró profilaxis en el caso de este tipo de contactos ya que el nivel de conocimientos del personal de la salud era suficiente.

En un estudio realizado en Estambul, se informó que solamente el 16,8\% de los animales con dueño habían sido vacunados. ${ }^{9}$ Şengöz, et al. detectaron que el 30\% de los animales tenían dueño, y el $6 \%$ habían sido vacunados. ${ }^{10}$ En forma similar, en nuestro estudio, el $23 \%$ de los animales tenían dueño y la proporción de animales con dueño y vacunados fue baja (11\%). Una proporción baja de vacunación en los animales con dueño se consideró indicador de conocimiento insuficiente sobre este tema por parte de los dueños de los animales de Turquía. Aumentar las tasas de vacunación de los animales con dueños por medio de capacitaciones públicas y medios audiovisuales nacionales permitiría reducir el volumen de trabajo y los costos relacionados con la profilaxis antirrábica.

La rabia puede infectar a los seres humanos mediante mordeduras, rasguños, contacto con materiales infectados, como saliva o tejido cerebral, una herida abierta, laceración o mucosas, inhalación o trasplante de órganos (córnea, etc.). ${ }^{16-18}$ Los resultados de nuestro estudio son similares a los de la bibliografía: el 58\% de los casos ingresaron por una mordedura, el $40 \%$, por un rasguño, el $2 \%$ por contacto con una herida abierta; se halló que el índice de mordeduras era estadística y significativamente más alto en comparación con otros tipos de contacto.

El lugar de la herida debido a una mordedura de perro o gato varía según el tipo de animal y la edad del sujeto. Sin embargo, se informó que las extremidades son el lugar de herida más frecuente. Se observaron lesiones en la cabeza y el cuello en el 9,5\% de los casos, en las extremidades inferiores en el 36,1\%, y en las extremidades superiores en el 30,4\%. ${ }^{19}$ En nuestro estudio, la mitad de los casos correspondía a lesiones en las extremidades superiores. Se considera que los animales alcanzan las extremidades superiores de los niños debido a su corta estatura en comparación con los adultos.

Al analizar el momento del ingreso, se detectó que el $64 \%$ de los casos ingresaron al consultorio de emergencias dentro de las primeras 24 horas. Si bien la muestra de este estudio está formada por residentes de áreas urbanas, este resultado es importante para indicar que aumentó la sensibilidad de la comunidad en contra del presunto contacto y de la rabia.

El tratamiento local de la herida en el período inicial después de la exposición es de vital importancia. Lavar la herida con agua y jabón de inmediato es el método más eficaz de protección contra la rabia. ${ }^{20}$ En todos nuestros casos, el lugar de la herida se lavó con agua y jabón para prevenir el ingreso del virus al tejido nervioso. La vacunación es el segundo paso más importante. ${ }^{13}$ Se administraron cinco dosis de la vacuna al $85 \%$ de los casos, ya que no fue posible realizar el seguimiento del animal, y tres dosis de la vacuna al $15 \%$ restante. La administración de concentrado de inmunoglobulinas antirrábicas es sumamente importante. ${ }^{21}$ Se halló que la proporción de administración de concentrado de inmunoglobulinas antirrábicas era bajo en los estudios realizados en Turquía. ${ }^{7,8,10}$ Se administró concentrado de inmunoglobulinas antirrábicas al $6 \%$ de los casos de nuestro estudio, lo que coincide con los datos bibliográficos. Esta proporción baja podría ser consecuencia de que la mayoría de las lesiones son superficiales y de errores en la administración de la profilaxis postexposición.

En los estudios realizados en otros países, se informó que la proporción de adecuación era del $60-98 \%{ }^{23,24}$ En nuestro estudio, la profilaxis postexposición adecuada se aplicó en el 85,5\% de los casos, y se consideró inadecuada en el 14,5\%. 
Los errores se describen a continuación: iniciar la vacunación antes de completar el tiempo de observación del animal vacunado $(41,4 \%)$, iniciar la vacunación antes de completar el tiempo de observación del animal no vacunado del cual no se sospecha que tenga rabia $(51,7 \%)$, y no administrar concentrado de inmunoglobulinas antirrábicas aunque el animal se haya escapado o haya sido sacrificado (6,9\%). Este resultado indica que es necesario mejorar continuamente los conocimientos del personal de la salud mediante capacitación en el puesto de trabajo.

Las reacciones alérgicas son los efectos secundarios más frecuentes de la vacuna antirrábica. ${ }^{19}$ En nuestro estudio, no se notificaron efectos secundarios relacionados con la vacuna antirrábica o el concentrado de inmunoglobulinas antirrábicas.

En la bibliografía, ${ }^{25}$ se informó el caso de una niña de 15 años que sobrevivió después de desarrollar rabia un mes después de ser mordida por un murciélago. El tratamiento incluyó la inducción del coma mientras se esperaba la maduración de la respuesta inmunitaria innata; no se administró la vacuna antirrábica. Sin embargo, la supervivencia de una sola paciente no modifica las estadísticas avasalladoras de la rabia, que tiene el índice de letalidad más alto entre las distintas enfermedades infecciosas.

La mayor limitación de este estudio es que el análisis fue retrospectivo en un centro de vacunación antirrábica.

\section{CONCLUSIÓN}

Nuestro estudio indica que, lamentablemente, nuestro país sigue siendo parte de los países en vías de desarrollo en cuanto a la protección y el control de la rabia. En Turquía la rabia continúa siendo un problema de salud pública grave, pero es solamente la punta del iceberg; y la carga económica del aumento de las tasas de vacunación como resultado de un control insuficiente de los animales es un punto a tener en cuenta.

En conclusión, consideramos que es necesario sensibilizar a la sociedad sobre la rabia y aumentar la cantidad de centros de vacunación antirrábica a los que puedan acceder fácilmente las personas de las áreas rurales; las autoridades locales deben ser más eficaces en el control de los animales callejeros, y estos servicios deben estar supervisados; la capacitación del personal de la salud en el puesto de trabajo es fundamental para mantener sus conocimientos actualizados.

\section{REFERENCIAS}

1. Rupprecht CE, Hanlon CA, Hemachudha T. Rabies reexamined. Lancet Infect Dis 2002;2(6):327-43.

2. Koruk ST, Ün H, Gürsoy B, Ünal N, Ç, et al. A human rabies case with antemortem diagnosis. Mikrobiyol Bul 2010;44(2):303-9.

3. Ünal H. Kuduz profilaksisi ve tedavici. Disponible en: http://www.infeksiyon.org/images/file_upload/ c2aad159311c682fbfeba08ee7782141Z847K5.pdf. [Fecha de acceso: 30 de julio de 2014].

4. Rabies Bulletin Europe 2013;37(2) Disponible en: http://www.who-rabies-bulletin.org/journal/Archive/ Bulletin_2013_2.pdf [Fecha de acceso: 30 de julio de 2014].

5. Sa lık Bakanlı 1 Temel Sa lık Hizmetleri Genel Müdürlü ü, Kuduz Korunma ve Kontrol Yönergesi. 09.05.2001,B100TSH011002/7755. Disponible en: http:/ / www.istanbulsaglik.gov.tr/w/mev/mev_yonr/y_kuduz_ kont_kor_y.pdf [Fecha de acceso: 12 de junio de 2015]

6. Özsoy M, Yakı tıran S, Özkan E. 2000 Yılında Kuduz A 1 Merkezine Ba vuran Hastaların De erlendırilmesi Evaluation Of The Patients Admitted To Rabies Vaccination Department In 2000. Türk Hij Den Biyol Derg 2002;59:1-6

7. Söğüt Ö, Sayhan MB, Gökdemir MT, Kara HP. Türkiye'nin Güneydo usunda Önlenebilir Bir Halk Sa 111 Sorunu: Kuduz Riskli Temas Olguları. JAEM 2011;10:14-7.

8. Temiz H, Akkoç H. Diyarbakır Devlet Hastanesi Kuduz Aş1 Merkezine Ba vuran 809 Olgunun De erlendirilmesi. Dicle Tip Dergisi 2008;35(3):181-4.

9. Dodet B, Goswami A, Gunasekera A, de Guzman F, et al. Rabies awareness in eight Asian countries. Vaccine 2008;26(50):6344-8.

10. Gökta P, Ceran N, Karagül E, Çiçek G, Özyürek S. Kuduz A i Merkezine Ba vuran 11017 Olgunun De erlendirilmesi. Klimik Dergisi 2002;15(1):12-5.

11. Sengoz G, Yasar KK, Karabela SN, Yildirim F, et al. Evaluation of cases admitted to a center in Istanbul, Turkey in 2003 for rabies vaccination and three rabies cases followed up in the last 15 years. Jpn J Infect Dis 2006;59(4):254-7.

12. Bleck TP, Rupprecht CE. Rhabdoviruses. In Mandell GL, Bennett JE, Dolin R. Principles and Practice of Infectious Diseases. 6th ed. Philadelphia: Churchill Livingstone; 2004:2047-56.

13. Kanra G, Kara A. Kuduz, patogenez, tani ve proflaksi. Hacettepe Tip Dergisi 2001;32(2):114-24.

14. Buzgan T, Irmak H, Yilmaz GR, Toruno lu MA, Safran A. Epidemiology of human rabies in Turkey: 1992-2007. Turk J Med Sci 2009;39(4):591-597.

15. Baykam N, Namdar O, Erten Ö. Rabies disease. In Sa lik Bakanli i, Zoonotik Hastalıklar. Zoonotik Hastalıklar Hizmet çi E itim Modülü. Ankara: Ba aMatbaacılık; 2011:81-104.

16. Wyatt J. Rabies-update on a global disease. Pediatr Infect Dis J 2007;26(4):351-2.

17. American Academy of Pediatrics: Rabies. In Pickering LK ed. Red Book 2006. Report of the Committee on Infectious Diseases. 26th edition. Elk Grove Village, IL: American Academy of Pediatrics; 2006: 552-9.

18. Dietzschold B, Koprowski H. Rabies transmission from organ transplants in the USA. Lancet 2004, 364(9435):648-9.

19. Ostanello F, Gherardi A, Caprioli A, La Placa L, et al. Incidence of injuries caused by dogs and cats treated in 
emergency departments in a major Italian city. Emerg Med J 2005;22(4):260-2.

20. World survey of rabies, 1997. Wkly Epidemiol Rec1999;74(45):381-4.

21. Warell MJ. Rabies. In Cook GC edit. Manson's Tropical Diseases. 20th ed.Philadelphia:WBSaunders;1996:700-720.

22. Wilde H,ChutivongseS, Tepsumethanon W, Choomkasien P, et al. Rabies in Tailand: 1990. Rev Infect Dis1991;13(4): 644-52.

23. Tepsumethanon S, Tepsumethanon V, Tantawichien T,
Suwansrino K, Wilde H. Problems in human rabies post exposure prophylaxis management. Travel Med Infect Dis 2007;5(3):189-93.

24. Dubnov J,Hefer E, Rubin L, RishponS; Haifa District Health Office. A change in rabies post-exposure treatment guide lines after decision analysis in Israel. Eur J Public Health 2007;17(1):92-7.

25. Willoughby RE, Tieves KS, Hoffman GM, Ghanavem NS, et al. Survival after treatment of rabies with induction of coma. N Engl J Med 2005; 352(24): 2508-14.

Los hombres grandes son aquellos que sienten que lo espiritual es más poderoso que cualquier fuerza material y que son las ideas las que rigen el mundo. 